\title{
On the Uniqueness of Individual Demand at Almost Every Price System
}

\author{
WALTER TROCKEL* \\ Department of Economics, SFB 21, University of Bonn, West Germany
}

Received January 20, 1983; revised September 28, 1983

A simple new proof, based on Fubini's theorem, is given for the uniqueness of individual demand at almost every price system, even if preferences are nonconvex. Journal of Economic Literature Classification Number: 022.

\section{INTRODUCTION}

The fact that even in case of a nonconvex preference relation the demand set is a singleton at almost every budget situation has been proved by Mas-Colell [2] and by Mas-Colell and Neuefeind [3]. Mas-Colell's proof made use of the theory of Hausdorff measures on lower dimensional subspaces of a Euclidian space. The proof due to Mas-Colell and Neuefeind relies on an application of the projection theorem for analytic sets due to Marczewski and Ryll-Nardzewski [1] together with Fubini's theorem. In the present note I shall give a new proof which relies only on the disintegration theorem (cf. Parthasarathy, [4, Theorem 8.1, p. 147]), i.e., a general version of Fubini's theorem.

\section{RESULT}

Consider $l \geqslant 2$ perfectly divisible commodities. An agent is described by his consumption set $P$, the positive orthant of the commodity space $\mathbb{R}^{l}$, his preference relation $\geqslant$ a reflexive, transitive, continuous binary relation on $P$, and his wealth $w \in L \equiv(0, \infty)$. The preference relation $\geqslant$ is moreover

* A grant of the Deutsche Forschungsgemeinschaft under which this note was written is gratefully acknowledged. 
assumed to be weakly monotone, i.e., $\left(\forall i \in\{1, \ldots, l\}: x_{i}>y_{i}\right) \Rightarrow(x>y)$. The space of normalized price systems $p$ is $L^{l-1}$. Denote by $\lambda^{l-1}$ and $\lambda^{1}$ the Lebesgue measures on $\left(L^{l-1}, \mathscr{B}\left(L^{l-1}\right)\right)$ and on $(L, \mathscr{B}(L))$, respectively, and by $\# M$ the cardinality of the set $M$. The demand set of an agent, described by $(\succcurlyeq, w)$ at the price system $p$ is

$$
\varphi(\succcurlyeq, w, p)=\{x \in P \mid(y>x) \Rightarrow(p y>w)\},
$$

i.e., the set of $\geqslant$-maximal commodity bundles in his budget set.

Proposition. Let $\geqslant$ be a weakly monotone continuous preference relation on $P$. Then

$$
\lambda^{l-1} \times \lambda^{1}\left(\left\{(p, w) \in L^{l} \mid \# \varphi(\succcurlyeq, w, p)>1\right\}\right)=0 .
$$

Proof. By Fubini's theorem any measurable set of line segments in an $(l-1)$-dimensional cube is an $\lambda^{l-1}$-null set. Although only this is needed, in case $l=2$ the even stronger statement that a line can contain at most countably many disjoint segments is well known. To simplify notation we choose in the following $l=2$ without loss of generality.

Let $\mu$ be a probability on $\left(L^{2}, \mathscr{B}\left(L^{2}\right)\right)$ which is equivalent to $\lambda^{2}$, i.e., which has the same null sets as $\lambda^{2}$. Let the utility function $u$ represent $\geqslant$ and let $v$ be the indirect utility function defined by

$$
v: L^{2} \rightarrow L:\left(p_{1}, w\right) \mapsto u(\varphi(\geqslant, w, p)) .
$$

The map $v$ is a continuous, hence measurable map onto its image. Therefore, since $L^{2}$ and image $(v)$ are Polish spaces, $\mu$ has a disintegration

$$
\mu=\int_{L} \zeta_{t} \mu \circ v^{-1}(d t)
$$

where the probabilities $\zeta_{t}$ on $L^{2}$ live on the fibres $v^{-1}(t), t \in$ image $(v)$. One can easily derive that $\zeta_{t}$ is equivalent to $\lambda^{2}$, hence atomless for $\mu \circ v^{-1}$-almost every $t \in L$. This follows from the translation invariance of the Lebesgue measure $\lambda^{2}$.

Denote by $N$ the measurable set of pairs $\left(p_{1}, w\right) \in L^{2}$ with $\# \varphi(\geqslant, w, p)>1$. For any $t \in \operatorname{image}(v)$ the set $N \cap v^{-1}(t)$ can be at most countable, since an indifference curve can have at most countably many disjoint line segments. Therefore we get

$$
\lambda^{2}(N)=\mu(N)=\int_{L} \zeta_{t}(N) \mu \circ v^{-1}(d t)=\int_{L} \zeta_{t}\left(N \cap v^{-1}(t)\right) \mu \circ v^{-1}(d t)=0 .
$$




\section{REFERENCES}

I. E. Marczewski and C. Ryll-Nardzewski, Projections in abstract sets, Fund. Math. 40 (1953), 160-164.

2. A. MAs-Colell, A remark on a smoothness property of convex, complete preorders. $J$. Math. Econom. 3 (1976), 103-105.

3. A. MAs-Colell and W. NeuEfEInd, Some generic properties of aggregate excess demand and an application, Econometrica 45 (1977), 591-599.

4. K. R. Parthasarathy, "Probability Measures on Metric Spaces," Academic Press, New York, 1967. 